freely moved about so as to remain in contact with the surface.

To avoid misunderstanding, it should be said that Riemann's expression for $d s^{2}$ is not the only one that is taken to be the typical or standard formula. The important thing is that, given any formula for $d s^{2}$, in a $\mathrm{P}_{n}$, we can, by direct calculation, find an expression for the curvature of $P_{n}$ in the neighbourhood of any assigned point $(x)$. It is only when this curvature is everywhere the same that we have a $P_{n}$ for which the axiom of free mobility is valid. When the curvature varies from place to place we are not entitled, for instance, to assume that we can carry about an invariable foot-rule for purposes of physical measurement.

In the simpler theory of relativity we have a formula

$$
d s^{2}=d x^{2}+d y^{2}+d z^{2}-c^{2} d t^{2}, \ldots
$$

where $c$ is a real constant. As it originally presents itself, $x, y, z$ are ordinary rectangular coordinates, $t$ is the time, and $c$ the experimental velocity of light. By a suitable choice of units we can make the value of $c$ any finite constant that we please. Following Minkowski, I shall call $(x, y, z, t)$ a world-point; the aggregate of these points may be provisionally called a space-time world $\mathrm{P}(x, y, z, t)$.

When $t=t_{0}$, a constant, $d t=0$ and (I) reduces to the ordinary Euclidean formula. We may express this by saying that the sub-world $\mathrm{P}\left(x, y, z, t_{0}\right)$ is Euclidean. Actual experiments take time; so we cannot verify this assertion by observation. If, however, two observers, at different places, make measurements which begin and end at the same instants, we may expect their results to be consistent. As Prof. Einstein has pointed out, the question of simultaneity (and, indeed, of time itself, as an observed quantity) is a more difficult one than appears at first sight.

The main difficulty about (r), as it seems to me, is that the expression on the right is not a definite form ; hence in the neighbourhood of every "real" point $(x, y, z, t)$ there is a real region for which $d s^{2}$ is negative. It is possible that the difficulty of interpretation is more apparent than real, as is the case in some well-known examples. For instance, a hyperbola may be analytically defined as an ellipse of semiaxes $a, b i$, where $a, b$ are real; and, moreover, v. Staudt's theory of involution gives an actual geometrical meaning to the algebraic definition.

If, with $i^{2}=-\mathrm{I}$, we put $c t=i \tau$, the formula (I) becomes

$$
d s^{2}=d x^{2}+d y^{2}+d z^{2}+d \tau^{2}, \ldots
$$

the typical formula for a Euclidean $\mathrm{P}_{4}$. This makes it very tempting to assume that the successions of phenomena in our world of experience are, so to speak, sections of a space-rworld $\mathrm{P}(x, y, z, \tau)$, obtained by giving $\tau$ purely imaginary values. This point of view has been taken by Minkowski and others.

The mathematical theories of abstract geometry and kinematics are so complete that physicists have a definite set of hypotheses from which to choose the one most suited to their purpose; and besides this they have to frame axioms and definitions about time, energy, etc., with which the pure mathematician is not concerned.

Whatever may be the ultimate form given to the theory of relativity, the predictive quality of its formulæ gives it a high claim to attention, and it certainly seems probable that, for the sake of what Mach calls economy of thought, we may feel compelled to change our ideas of "actual " space and time.

In an article like this it is impossible to go into detail; the following references may be useful to readers who desire further information:-"The Elements of Non-Euclidean Geometry," by J. L. Coolidge, is rather condensed, but very conscientious and trustworthy; one of the best analytical discussions of the metrical theory is in Bianchi's "Lezioni di Geometria Differenziale," chap. xi.; and Lie's "Theorie der Transformationsgruppen," vol. iii., chaps. xx.-xxiv., contains a most valuable critique of Riemann and Helmholtz. The article "Geometry" in the "Encyclopædia Britannica" (last edition) gives an outline of the theory and numerous references. Finally, there is an elaborate "Bibliography of NonEuclidean Geometry " by D. M. J. Somerville (see NAture, May 16, r9i2, vol. Ixxxix., p. 266).

\title{
The General Physical Theory of Relativity.
}

\author{
By J. H. Jeans, Sec. R.S.
}

THE relativity theory of gravitation, which is at present the centre of so much interest, owes its existence to an earlier physical theory of relativity which had proved to be in accord with all the known phenomena of Nature except gravitation. The gravitational theory is only one branch, although a vigorous and striking branch, of a firmly established parent tree. The present article will deal solely with the main trunk and roots of this tree.

Newton's laws of motion referred explicitly to a state of rest, but also showed that the phenomena to be expected from bodies in a state of rest No. 2677 , voL. IO6] were precisely identical with those to be expected when the same bodies were moving with constant velocity. Indeed, Newton directed special attention to this implication of his laws of motion in the following words :-

COROLlary V.: The motions of bodies included in a given space are the same among themselves, whether that space is at rest, or moves uniformly forwards in a right line without any circular motion.

"A clear proof of which we have," continues Newton, "from the experiment of a ship, where all motions happen after the same manner whether 
the ship is at rest, or is carried uniformly forward in a right line."

Thus no experiment on board ship can ever disclose the ship's velocity through the sea. The matter stands differently to one who is free to experiment with both the ship and the sea. Let a sailor walk to the end of the bowsprit and drop his lead into the sea. A circular ripple will spread out; but every sailor knows that the point at which his line enters the wate w will not remain at the centre of this circle. The velocity with which the point of entry advances from the centre of the circle will give the velocity of the ship through the sea.

If our earth is ploughing its way through a sea of æther, an experiment conceived on similar lines ought to reveal the velocity of the earth through the æther. The famous Michelson-Morley experiment was designed to this end. Our earth was the ship; the physical laboratory at Chicago was the bowsprit. The dropping of the lead into the sea was represented by the emission of a lightsignal, and the wave-front emanating from this signal was the ripple on the sea of æther. In the original experiments of Michelson and Morley it was not possible to watch the progress of the ripple directly, but sufficient information was obtained by arranging mirrors to reflect the signal back to the starting-point. In the recent experiments of Majorana this difficulty is obviated, although at the cost of some loss of refinement.

From these and other experiments the result invariably emerges that the wave-front appears to be a sphere having the observer at its centre. Thus on the hypothesis that our earth is surrounded by a sea of æther, experiment shows that the velocity of the earth relative to this sea of æther is nil. We cannot suppose that the true velocity is always nil, for the earth is known to be describing circles around the sun at a speed of $30 \mathrm{~km}$. a second, while the experiments were sensitive enough to detect a velocity of onehundredth part of this.

In view of the complete success which has attended the hypothesis of relativity, it would scarcely seem to be necessary to do more than mention the various early hypotheses put forward to account for these and similar experimental results. Such were the hypotheses that the earth drags the æther along with it (Arago, 1818); that matter moving through the æther is contracted, as a result of its motion, in just such a way as eternally to conceal the earth's motion through the æther from our measurements (FitzGerald, 1893; Lorentz, 1895); and that light is a phenomenon of corpuscular emission (Ritz). Each of these hypotheses explained some only of the facts to be explained, and failed with others.

The theory of light has progressed largely through the construction of mechanical models. Every such model, if fruitful, suggests new laws to be tested. So long as the laws suggested in this way are confirmed by observation, the model stands; as soon as a predicted law is found to fail, the model must be amended or abandoned. No. 2677 , VOL. I06]
Notable examples of such models have been the corpuscular model of Newton, the elastic-solid æther of Young and his followers, and the electromagnetic æther of Faraday and Maxwell. The first two of these have long ago served their purpose and passed away. The time has now clearly come when the last of these optical models, the electromagnetic æther, must be either amended or abandoned, and the indications are strong that the less drastic course will not suffice.

The construction of mechanical models is not, however, the only known means of guidance to the discovery of new laws of Nature. An even more fruitful means of progress has been provided by tentative generalisation of known laws. Proved laws $a, b, c, d \ldots$ are found to be special cases of a more general law $\mathrm{A}$, and the truth of $\mathrm{A}$ is then seen to involve not only the detailed laws $a, b, c, d \ldots$ which have led to $\mathrm{A}$, but also other detailed laws $p, q, r, s \ldots$ In this way we may be guided to test the suggested new laws $p, q, r$, $s$...., and the generalisation $\mathrm{A}$ is, of course, strengthened or discredited according as $p, q, r, s$ . . . are confirmed by observation or not. Conspicuous instances of successful generalisations of this kind are provided by the conservation of energy and the second law of thermodynamics.

Early in the present century Einstein and Lorentz suggested a tentative generalisation of this type, which is now known as the hypothesis of relativity. Since all experiments so far performed had failed to disclose the velocity of the earth through the assumed æther, it was natural to generalise in the first place to the tentative principle that this velocity could not, from the nature of things, ever be revealed by any experiment whatever. Generalised somewhat further by the removal of the local reference to our earth, the hypothesis assumed the form that all phenomena of Nature are the same for an observer moving with any uniform velocity as they are for an observer at rest. This somewhat crude form of statement shows that the hypothesis merely generalises Newton's corollary V. quoted above, so as to make it apply to all the phenomena of Nature. Since, however, the acceptance of the hypothesis makes it impossible to define what is meant by a state of rest, it is better to express the hypothesis in the form that all the phenomena of Nature are the same for any two observers who move relative to one another with a constant velocity.

This hypothesis is known already to be true as regards the mechanical forces considered in Newton's laws. Naturally, also, it is true as regards the optical phenomena investigated in the Michelson-Morley and similar experiments, for it is out of these phenomena that the hypothesis arose. The crucial test occurs when laws in other fields of science are deduced from the hypothesis and compared with observation. The hypothesis has been very thoroughly tested in the field of electromagnetism, and in every single case has emerged triumphant. As conspicuous instances of its success may be mentioned: The explanation 
of Fizeau's water-tube experiment, the prediction of the law connecting electronic mass with velocity, and the prediction of ponderomotive electromagnetic forces in moving media.

One final, and therefore crucial, test remains : gravitation. It was soon noticed that the hypothesis was inconsistent with the exact truth of Newton's gravitational law of force $m m^{\prime} / r^{2}$. Thus the hypothesis of relativity predicts that a freely moving planet cannot describe a perfect ellipse about the sun as focus. This prediction is made on quite general grounds, just as the conservation of energy predicts that a stream of water cannot flow uphill. But the conservation of energy by itself is powerless to predict what will be the actual course of a stream of water, and in precisely the same way the hypothesis of relativity alone is powerless to predict what will be the orbit of a planet. Before this or any other positive gravitational predictions can be made, additional hypotheses must be introduced. The main trunk of the tree is the relativity hypothesis already mentioned; these additional hypotheses form the branches. The trunk can exist without its branches, but not the branches without the trunk. Whether the branches have been placed on the trunk with complete accuracy is admittedly still an open question-it must of necessity remain so until the difficult questions associated with the gravitational shift of spectral lines have been finally settled-but the main trunk of the tree can be disturbed by nothing short of a direct experimental determination of the absolute velocity of the earth, and the only means which can possibly remain available for such a determination now are gravitational.

\section{The Michelson-Morley Experiment and the Dimensions of Moving Bodies.}

\section{By Prof. H. A. Lorentz, For.Mem.R.S.}

A doubts have sometimes been expressed concerning the interpretation of Prof. Michelson's celebrated experiment, some remarks on the subject will perhaps not be out of place here. I shall try to show, by what seems to me an unimpeachable mode of reasoning, that, if we adopt Fresnel's theory of a stationary æther, supposing also that a material system can have a uniform translation with constant velocity $v$ without changing its dimensions, we must surely expect the result that was predicted by Maxwell.

Let us introduce a system of rectangular axes of co-ordinates fixed to the material system, the axis of $x$ being in the direction of the motion. Then, with respect to these axes, the æther will flow with the velocity $-v$. The progress of waves of light, relatively to them, may be traced by means of Huygens's principle; for this purpose it suffices to know the form and position of the elementary waves. For the sake of generality I shall suppose the propagation to take place in a material medium of refractive index $\mu$, so that, if $c$ is the velocity of light in the æther, the velocity in the medium when at rest would be $c / \mu$. The elementary wave formed in the time $d t$ around a point $\mathrm{P}$ will be a sphere of radius $(c / \mu) d t$, of which the centre $\mathrm{P}^{\prime}$ does not, however, coincide with $\mathrm{P}$, the line $\mathrm{PP}^{\prime}$ being in the direction opposite to that of $\mathrm{OX}$, and having the length $\left(v / \mu^{2}\right) d t$ (Fresnel's coefficient).

If $Q$ is any point on the surface of the sphere, $P Q$ can be considered as an element of a ray of light, and $w=\mathrm{PQ} / d t$ will be the velocity of the ray. Confining ourselves to terms of the second order, i.e. of the order $v^{2} / c^{2}$, and denoting by $\delta$ the angle between the ray and $\mathrm{OX}$, we have

$$
\frac{\mathrm{I}}{w}=\frac{\mu}{c}+\frac{v}{c^{2}} \cos \delta+\frac{v^{2}}{2 \mu c^{3}}\left(\mathrm{I}+\cos ^{2} \delta\right) .
$$

Now, let $\mathrm{A}$ and $\mathrm{B}$ be points having fixed positions in the material system. The course $s$ of a ray of light passing from $A$ to $B$ will be determined by the condition that the integral

$$
\int \frac{d s}{w}
$$

is a minimum. Using the above value of $1 / w$, it is easily shown that, if quantities of the second order are neglected, the course of the ray is not affected by the translation $v$, so that, if $L_{0}$ is the path of the ray in the case $v=0$, and $L$ the real path, these lines will be distant from each other to an amount of the second order only. Hence, if in the case of a translation $v$ we calculate by means of ( 1 ) the integral (2), both for $L$ and $L_{0}$, the two values will differ by no more than a quantity of the fourth order; indeed, since the integral is a minimum for $\mathrm{L}$, the difference must be of the second order with respect to the distances between $L$ and $L_{0}$, and these distances are already of the second order of magnitude.

It is seen in this way that, so long as we neglect terms of an order higher than the second, we may replace

$$
\int_{i} \frac{d s}{w} \text { by } \int_{L_{0}} \frac{d s}{w},
$$

an argument that must not be overlooked in the theory of the experiment. On the ground of it we shall commit no error if, in determining the paths $L_{1}$ and $L_{2}$ of two rays that start from a point $A$, and are made to interfere at a point $B$, we take no account of the motion of the apparatus. The change in the difference of phase produced by the translation will be given by the difference between the values which the integral

$$
\int \frac{z^{2}}{2 \mu c^{3}}\left(\mathrm{I}+\cos ^{2} \delta\right) d s
$$

takes for the lines $L_{1}$ and $L_{2}$ so determined. If, along the first of them, $\cos ^{2} \delta=1$, and along the 